\title{
Erratum to: Management of soil nitrate heterogeneity resulting from crop rows in a lettuce-tomato rotation under a greenhouse
}

François Lecompte

Published online: 21 December 2011

(C) INRA and Springer-Verlag, France 2011

Erratum to: Agron. Sustain. Dev.

DOI 10.1007/s13593-011-0047-8

In the original article Table 3 is not correct. The calculated values of leaching for the "L" system is $54 \mathrm{~kg} / \mathrm{ha}$ in the inter-row (IR), and $25 \mathrm{~kg} / \mathrm{ha}$ in the row (R), and NOT 54 and $75 \mathrm{~kg} / \mathrm{ha}$ as published in the online version of the article. We reprint the complete corrected table here below.

Everything else in the paper remains correct.

The online version of the original article can be found at http://dx.doi. org/10.1007/s13593-011-0047-8.

F. Lecompte $(\bowtie)$

UR 1115 Plantes et Systèmes de Cultures Horticoles, INRA,

Domaine Saint Paul, Site Agroparc,

84914, Avignon Cedex, France

e-mail: francois.lecompte@avignon.inra.fr 
Table $3 \mathrm{~N}$ balance components for lettuce at two positions (Row $\mathrm{R}$ and Inter-row IR) and for two management systems "C"

(current farm practices) and " $\mathrm{L}$ " (limited water and nutrient inputs)

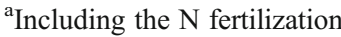
before lettuce transplanting in system $\mathrm{C}$

${ }^{b}$ Estimated from stock variations between September and November 2008

${ }^{\mathrm{c}} L=M-U-\left(S_{\mathrm{f}}-S_{\mathrm{i}}\right)$. Part of this term may include unaccounted denitrification or volatilization

\begin{tabular}{|c|c|c|c|c|c|}
\hline & \multirow[t]{2}{*}{$\mathrm{kgN} / \mathrm{ha}$} & \multicolumn{2}{|l|}{$\mathrm{C}$} & \multicolumn{2}{|l|}{$\mathrm{L}$} \\
\hline & & IR & $\mathrm{R}$ & IR & $\mathrm{R}$ \\
\hline \multirow[t]{6}{*}{ 2006-2007 (L1) } & Initial stock $\left(S_{\mathrm{i}}\right)^{\mathrm{a}}$ & 160 & 106 & 59 & 34 \\
\hline & Final stock $\left(S_{\mathrm{f}}\right)$ & 30 & 16 & 13 & 10 \\
\hline & $S_{\mathrm{f}}-S_{\mathrm{i}}$ & -130 & -90 & -46 & -24 \\
\hline & Crop uptake $(U)$ & 99 & 76 & 58 & 38 \\
\hline & Mineralization $(M)^{\mathrm{b}}$ & 39 & 39 & 39 & 39 \\
\hline & Leaching $(L)^{\mathrm{c}}$ & 70 & 53 & 27 & 25 \\
\hline \multirow[t]{6}{*}{ 2007-2008 (L2) } & Initial stock $\left(S_{\mathrm{i}}\right)^{\mathrm{a}}$ & 156 & 96 & 78 & 34 \\
\hline & Final stock $\left(S_{\mathrm{f}}\right)$ & 25 & 21 & 19 & 18 \\
\hline & $S_{\mathrm{f}}-S_{\mathrm{i}}$ & -131 & -75 & -59 & -16 \\
\hline & Crop uptake $(U)$ & 87 & 63 & 47 & 33 \\
\hline & Mineralization $(M)^{\mathrm{b}}$ & 42 & 42 & 42 & 42 \\
\hline & Leaching $(L)^{\mathrm{c}}$ & 86 & 54 & 54 & 25 \\
\hline
\end{tabular}

